\title{
Synthesis and bioactive evaluations of novel benzotriazole compounds as potential antimicrobial agents and the interaction with calf thymus DNA
}

\author{
YU REN, HUI ZHEN ZHANG, SHAO LIN ZHANG, YUN LEI LUO, LING ZHANG, \\ CHENG HE ZHOU and RONG XIA GENG* \\ School of Chemistry and Chemical Engineering, Southwest University, Chongqing 400715, \\ People's Republic of China \\ e-mail: geng0712@swu.edu.cn
}

MS received 16 April 2015; revised 12 September 2015; accepted 5 October 2015

\begin{abstract}
A novel series of benzotriazole derivatives were synthesized and characterized by NMR, IR and MS spectra. The bioactive assay manifested that most of the new compounds exhibited moderate to good antibacterial and antifungal activities against the tested strains in comparison to reference drugs chloromycin, norfloxacin and fluconazole. Especially, 2,4-dichlorophenyl substituted benzotriazole derivative $\mathbf{6 f}$ displayed good antibacterial activity against MRSA with MIC value of $4 \mu \mathrm{g} / \mathrm{mL}$, which was 2-fold more potent than Chloromycin, and it also displayed 3-fold stronger antifungal activity (MIC $=4 \mu \mathrm{g} / \mathrm{mL}$ ) than fluconazole (MIC $=16 \mu \mathrm{g} / \mathrm{mL}$ ) against Beer yeast. The preliminary interactive investigations of compound $\mathbf{6} \mathbf{f}$ with calf thymus DNA revealed that compound $\mathbf{6} \mathbf{f}$ could effectively intercalate into DNA to form compound $\mathbf{6} \mathbf{f}-\mathrm{DNA}$ complex which might block DNA replication to exert antimicrobial activities. Molecular docking experiments suggested that compound $\mathbf{6 f}$ projected into base-pairs of DNA hexamer duplex forming two hydrogen bonds with guanine of DNA. The theoretical calculations were in accordance with the experimental results.
\end{abstract}

Keywords. Benzotriazole; antibacterial; antifungal; calf thymus DNA interactions; molecular docking.

\section{Introduction}

Bacterial and fungal infections have become continual and growing threats to human health in the past few decades. ${ }^{1-3}$ Although large numbers of antibiotics and synthetic drugs such as sulfonamides, quinolones, oxazolidones and azoles are widely used, the sensibility of currently available drugs have remarkably decreased for a variety of pathogens and newly rising multidrug-resistant strains. ${ }^{4,5}$ Therefore, the discovery of new compounds with remarkable antibacterial and antifungal activities, broad antimicrobial spectrum, few adverse effects, low toxicity, especially with action mechanisms distinct from the well-known antimicrobial agents is of great importance. ${ }^{6}$

Fluconazole is a first-line oral triazole-antifungal drug recommended by World Health Organization (WHO), which has established an exceptional therapeutic record for Candida infections and has become the first choice for the treatment of Candida albicans and Cryptococcus neoformans due to favorable pharmacokinetic characteristics. ${ }^{7}$ It acts by coordinating with the iron(II) ion of heme to block the biosynthesis of

*For correspondence ergosterol, which is the essential component of the fungal cell membrane, thus destroying the integrity of the fungal cell wall and inhibiting the growth and breeding of fungi. ${ }^{8}$ However, this action generally leads to severe toxicity and limits its application in the treatment of deep-seated mycoses and life-threatening systemic infections. In addition, the extensive clinical use of fluconazole has resulted in the emergence of fluconazoleresistant Candida albicans isolates. ${ }^{9}$ Moreover, fluconazole with low water solubility is not effective against invasive aspergillosis. For these reasons, many recent researches have been devoted to further structural modification of fluconazole in order to broaden its antimicrobial spectrum and improve its therapeutic indices. ${ }^{10}$ In our previously reported work, we have developed novel structural fluconazole analogues, in which triazoles in the side chains were replaced by its bioisosteres, such as imidazole and benzimidazole (figure 1). ${ }^{11}$ Bioactive evaluation results suggested that this type of fluconazole analogues possessed good antimicrobial activities which were comparable to or even better than the reference drugs chloromycin, norfloxacin and fluconazole. ${ }^{12}$ Therefore, we have further investigated this type of fluconazole analogues as novel antimicrobial agents. 

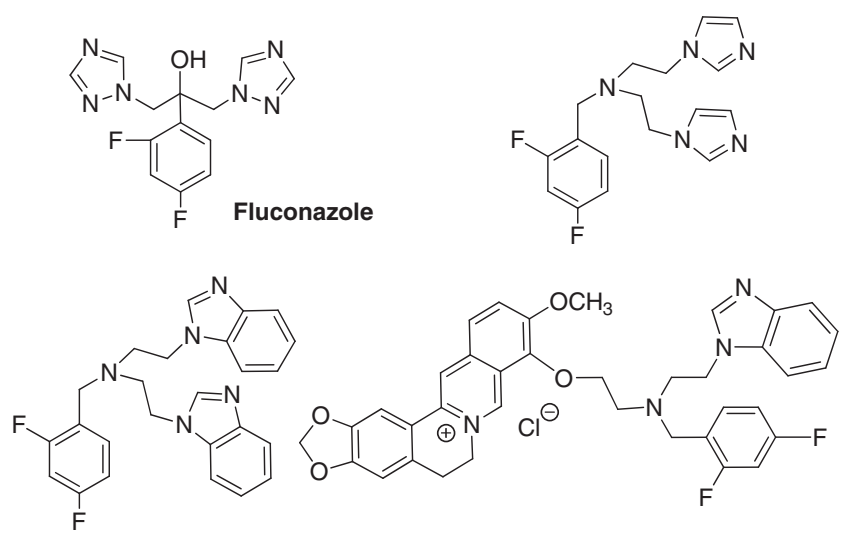

Figure 1. Imidazole and benzimidazole analogues of fluconazole reported by our group.

Benzotriazole is a fused aromatic nitrogen heterocycle of benzene ring with triazole and is an important bioisostere of triazole ring. ${ }^{13-16}$ Compared with triazole, the fused benzene ring and the threenitrogen structure make benzotriazole nucleus possess a larger conjugated system and better electronic transfer property, thereby more readily binding with DNA, enzymes and receptors in organisms via diverse non-covalent interactions like hydrogen bonds, coordination bonds and $\pi-\pi$ stacking interactions, thus resulting in a broad spectrum of biological activities such as anticancer, ${ }^{17-21}$ antifungal, ${ }^{22,23}$ antibacterial, ${ }^{24,25}$ antitubercular, ${ }^{26}$ antiviral, ${ }^{27}$ antiparasitic, ${ }^{28}$ and antioxidative activities. ${ }^{29,30}$ Recent literature evidences indicate that the combination of benzotriazole and tertiary alcohol frame could afford novel fluconazole analogues with considerable antimicrobial activities. For example, benzotriazole derivative PDP displayed excellent antifungal activity against both Candida albicans (ATCC90028) and Candida parapsilosis (ATCC 22019) with MIC value of $0.8 \mu \mathrm{g} / \mathrm{mL}$, which was equivalent to that of fluconazole. ${ }^{31}$ Moreover, another structural similar benzotriazole derivative YGP gave good antifungal activity against Candida arachidicoa with inhibition percentage of $62.7 \%$, which was stronger than the commercial fungicide difenoconazole (figure 2). ${ }^{32}$ These evidences clearly point out that benzotriazole derivatives possess large potential as new antimicrobial agents. For these reasons, benzotriazole alcohol type of fluconazole analogues have attracted our special interest.

Based on the above considerations, we introduced benzotriazole ring into the tertiary alcohol type of fluconazole analogue skeleton to replace five-membered triazole ring with the aim to investigate the effect of benzene-fused triazole ring on biological activities. The piperazine was incorporated into the target compounds

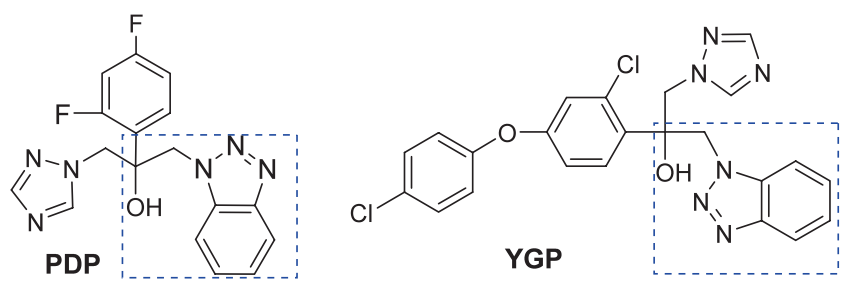

Figure 2. Structures of some antimicrobial benzotriazole tertiary alcohols.

since a lot of literature revealed that the introduction of piperzaine was an important synthetic strategy in drug discovery due to its easy modification, proper alkalinity, water solubility, capacity for the formation of hydrogen bonds and adjustment of molecular physicochemical properties. ${ }^{33}$ Furthermore, 2,4-dichlorobenzyl moiety was introduced to substitute 2,4-diflurophenyl group of fluconazole, which might enhance the rate of absorption and transport of drugs in vivo with enhanced lipid solubility, thus improving the pharmacological properties. A variety of substituted benzyl groups were also incorporated into target compounds to study their contributions to the antimicrobial activities. The newly synthesized benzotriazole derivatives might be expected to exhibit potentiality against bacterial and fungal strains, especially the drug-resistant microorganism. Therefore, all the new compounds were screened for antibacterial and antifungal activities in vitro and the preliminary structure-activity relationships (SAR) are discussed. The interaction of most active compound $\mathbf{6 f}$ with calf thymus DNA was also evaluted by UV-vis absorption spectroscopy on a molecular level to investigate its probable antimicrobial mechanism. Finally, molecular docking studies were employed to reconfirm the interaction behaviors between the prepared compound and DNA hexamer duplex.

\section{Experimental}

\subsection{General Methods}

Melting points, uncorrected, were recorded on X-6 melting point apparatus. TLC analysis was done using pre-coated silica gel plates. FT-IR spectra were carried out on Bruker RFS100/S spectrophotometer (Bio$\mathrm{Rad}$, Cambridge, MA, USA) using $\mathrm{KBr}$ pellets in the $400-4000 \mathrm{~cm}^{-1}$ range. NMR spectra were recorded on a Bruker AV 300 or Varian 400 spectrometer using TMS as an internal standard. The chemical shifts were reported in parts per million (ppm), the coupling constants $(J)$ were expressed in hertz $(\mathrm{Hz})$ and signals were described as singlet (s), doublet (d), triplet (t), as well as multiplet $(\mathrm{m})$. The following abbreviations were used to 
designate aryl groups: Btz, benzotriazolyl; Pip, piperazine; Mor, Morpholine; Ph, phenyl. The mass spectra were recorded on LCMS-2010A. The UV spectrum was recorded at room temperature on a TU-2450 spectrophotometer (Puxi Analytic Instrument Ltd. of Beijing, China) using $1.0 \mathrm{~cm}$ quartz cells. Calf thymus DNA and NR were obtained from Sigma-Aldrich (St. Louis, MO, USA). Tris-HCl and DMF were of analytical purity. NR stock solution $\left(2.0 \times 10^{-3} \mathrm{~mol} / \mathrm{L}\right)$ was prepared by dissolving its solid in doubly distilled water and was kept in a cool and dark place. All the solutions were adjusted with Tris- $\mathrm{HCl}$ buffer solution $(\mathrm{pH}=7.4)$, which was prepared by mixing and diluting Tris buffer solution with $\mathrm{HCl}$ solution. All other chemicals and solvents were commercially available, and were used without further purification.

\subsection{General procedures for the preparation of inter- mediates $\mathbf{2}, \mathbf{3}, \mathbf{5 a}-\boldsymbol{i}$}

The intermediates $\mathbf{2}, \mathbf{3}$ and $\mathbf{5 a}-\mathbf{i}$ were prepared according to previously reported methods. ${ }^{34,35}$

2.3 General procedures for the preparation of 2-((2(2,4-dichlorophenyl)oxiran-2-yl)methyl)-2H-benzo[d] [1,2,3]triazole (4)

To a solution of compound $3(5.000 \mathrm{~g}, 0.016 \mathrm{~mol})$ in toluene $(15 \mathrm{~mL})$ was added trimethylsulfoxonium iodide $(3.594 \mathrm{~g}, 0.016 \mathrm{~mol})$ followed by the addition of $20 \%$ sodium hydroxide solution $(2.5 \mathrm{~mL})$. The reaction mixture was then heated at $60^{\circ} \mathrm{C}$ for $6 \mathrm{~h}$. After the reaction was completed (monitored by TLC, ethyl acetate/light petroleum $(1: 1, \mathrm{v} / \mathrm{v}))$, it was diluted with ethyl acetate $(3 \times 20 \mathrm{~mL})$. The organic layer was washed with water $(2 \times 30 \mathrm{~mL})$, dried over anhydrous sodium sulfate. The filtrate was concentrated under reduced pressure and the residue was purified by flash silica gel column eluting with ethyl acetate/light petroleum $(1: 1, \mathrm{v} / \mathrm{v})$ to give compound $\mathbf{4}$ as white solid. Yield: $68.2 \%$; M.p.: $124-125^{\circ} \mathrm{C}$. IR $\left(\mathrm{KBr}, \mathrm{cm}^{-1}\right)$ : 3019 , 2930, 2861, 1618, 1584, 1502, 1457, 1333, 1269, 1103, 970, 828, 785, 679; ${ }^{1} \mathrm{H}$ NMR $\left(600 \mathrm{MHz}, \mathrm{CDCl}_{3}\right): \delta$ $8.02(\mathrm{~d}, 1 \mathrm{H}, J=8.4 \mathrm{~Hz}, \mathrm{Btz}-4-H), 7.64(\mathrm{~d}, 1 \mathrm{H}, J=$ $8.4 \mathrm{~Hz}, \mathrm{Btz}-7-H), 7.51-7.48$ (m, 1H, Ph-3-H), 7.357.39 (m, 2H, Btz-5,6-H), 7.03-7.01 (m, 1H, Ph-5-H), $6.92(\mathrm{~d}, 1 \mathrm{H}, J=8.3 \mathrm{~Hz}, \mathrm{Ph}-6-H), 5.46(\mathrm{~d}, 1 \mathrm{H}, J=$ $\left.15.2 \mathrm{~Hz}, \mathrm{Btz}-\mathrm{CH}_{2}\right), 4.84\left(\mathrm{~d}, 1 \mathrm{H}, J=15.2 \mathrm{~Hz}, \mathrm{Btz}-\mathrm{CH}_{2}\right)$, $3.07\left(\mathrm{~d}, 1 \mathrm{H}, J=4.6 \mathrm{~Hz}, \mathrm{CH}_{2}-\mathrm{O}\right), 2.93(\mathrm{~d}, 1 \mathrm{H}, J=$ $\left.4.6 \mathrm{~Hz}, \mathrm{CH}_{2}-\mathrm{O}\right) \mathrm{ppm} ;{ }^{13} \mathrm{C} \mathrm{NMR}\left(150 \mathrm{MHz}, \mathrm{CDCl}_{3}\right)$ : $\delta 145.92,135.56,133.73,133.45,132.87,130.16$, $129.31,127.73,127.42,124.05,120.00,109.97,59.12$, $52.15,52.01 \mathrm{ppm} ; \mathrm{MS}(\mathrm{m} / \mathrm{z}): 321.18[\mathrm{M}+\mathrm{H}]^{+}$.

\subsection{General procedures for the preparation of compounds $\mathbf{6 a}-\boldsymbol{i}$}

2.4a 1-(1H-benzo[d][1,2,3]triazol-1-yl)-2-(2,4-dichlorophenyl)-3-(4-(4-methoxybenzyl) piperazin-1-yl)propan2-ol (6a): To a solution of compound $4(500 \mathrm{mg}$, $1.56 \mathrm{mmol})$ in ethanol $(15 \mathrm{~mL})$ was added compound 5a $(322 \mathrm{mg}, 1.56 \mathrm{mmol})$ followed by the addition of sodium bicarbonate $(131 \mathrm{mg}, 1.56 \mathrm{mmol})$. The reaction mixture was stirred at $70^{\circ} \mathrm{C}$ for $18 \mathrm{~h}$. After the reaction was completed (monitored by TLC, methanol/ dichloromethane $(1 / 200, \mathrm{v} / \mathrm{v}))$, the reaction mixture was cooled to room temperature and treated with formic acid to adjust the $\mathrm{pH}$ value to 5.5-6.5. After the ethanol was removed under reduced pressure, the mixture was purified by flash silica gel column eluting with methanol/dichloromethane $(1 / 100, \mathrm{v} / \mathrm{v})$ to give the desired compound $\mathbf{6 a}$ as yellow liquid. Yield: $46.2 \%$. IR $\left(\mathrm{KBr}, \mathrm{cm}^{-1}\right)$ : 3440, 3112, 3029, 2986, 2840, 1602, 1587, 1512, 1458, 1347, 1235, 1141, 984, 857, 783, $681 \mathrm{~cm}^{-1} .{ }^{1} \mathrm{H}$ NMR $\left(600 \mathrm{MHz}, \mathrm{CDCl}_{3}\right): \delta 7.98(\mathrm{~d}, 1 \mathrm{H}$, $J=8.3 \mathrm{~Hz}, \mathrm{Btz}-4-H), 7.72(\mathrm{~d}, 1 \mathrm{H}, J=8.6 \mathrm{~Hz}, \mathrm{Btz}-7-$ $H), 7.73-7.71(\mathrm{~m}, 1 \mathrm{H}, \mathrm{C}-\mathrm{Ph}-3-H), 7.44-7.39$ (m, 2H, Btz-5,6-H), 7.33-7.30 (m, 1H, C-Ph-5-H), 7.23 (s, 1H, C-Ph-6- $H$ ), 7.14-7.12 (m, 2H, CH $-\mathrm{Ph}-2,6-H), 6.80$ $\left(\mathrm{d}, 2 \mathrm{H}, J=8.5 \mathrm{~Hz}, \mathrm{CH}_{2}-\mathrm{Ph}-3,5-H\right), 5.67(\mathrm{~s}, 1 \mathrm{H}, \mathrm{OH})$, $5.23\left(\mathrm{~d}, 1 \mathrm{H}, J=14.6 \mathrm{~Hz}, \mathrm{Btz}-\mathrm{CH}_{2}\right), 4.98(\mathrm{~d}, 1 \mathrm{H}, J=$ $\left.14.6 \mathrm{~Hz}, \mathrm{Btz}-\mathrm{CH}_{2}\right), 3.76\left(\mathrm{~s}, 3 \mathrm{H}, \mathrm{OCH}_{3}\right), 3.61(\mathrm{~d}, 1 \mathrm{H}$, $J=13.7 \mathrm{~Hz}, \mathrm{C}-\mathrm{CH}_{2}$-Pip), 3.33 (d, $2 \mathrm{H}, J=21.6 \mathrm{~Hz}$, Pip- $\mathrm{CH}_{2}-\mathrm{Ph}$ ), 2.85 (d, $1 \mathrm{H}, J=13.7 \mathrm{~Hz}, \mathrm{C}-\mathrm{CH}_{2}$-Pip), 2.35-2.31 (m, 4H, Pip), 2.25-2.16 (m, 4H, Pip) ppm; ${ }^{13} \mathrm{C} \mathrm{NMR}\left(150 \mathrm{MHz}, \mathrm{CDCl}_{3}\right): \delta 158.91,145.74,140.01$, $135.09,134.51,131.44,130.83,130.22,130.02$, $129.67,128.76,127.44,127.06,126.90,123.61$, $119.54,113.70,111.38,73.85,62.09,60.73,55.23$, 54.73, 54.02, 52.75 ppm; MS $(m / z): 527.74[\mathrm{M}+\mathrm{H}]^{+}$.

2.4b 1-(1H-benzo[d][1,2,3]triazol-1-yl)-3-(4-(3-chlorobenzyl)piperazin-1-yl)-2-(2,4-dichloro phenyl)propan2-ol $(\boldsymbol{6} \boldsymbol{b})$ : Yellow oil. Yield: $45.5 \%$. IR $\left(\mathrm{KBr}, \mathrm{cm}^{-1}\right)$ : 3447, 3112, 3029, 2986, 2870, 1610, 1588, 1509, 1460, $1353,1236,1137,973,866,785,680 \mathrm{~cm}^{-1} \cdot{ }^{1} \mathrm{H}$ NMR $\left(600 \mathrm{MHz}, \mathrm{CDCl}_{3}\right): \delta: 7.98(\mathrm{~d}, 1 \mathrm{H}, J=8.3 \mathrm{~Hz}, \mathrm{Btz}-4-$ $H), 7.73(\mathrm{~d}, 1 \mathrm{H}, J=8.6 \mathrm{~Hz}, \mathrm{Btz}-7-H), 7.72-7.70(\mathrm{~m}$, 1H, C-Ph-3-H), 7.46-7.39 (m, 2H, Btz-5,6-H), 7.33$7.31(\mathrm{~m}, 1 \mathrm{H}, \mathrm{C}-\mathrm{Ph}-5-H), 7.23\left(\mathrm{~s}, 1 \mathrm{H}, \mathrm{CH}_{2}-\mathrm{Ph}-2-H\right)$, $7.22(\mathrm{~s}, 1 \mathrm{H}, \mathrm{C}-\mathrm{Ph}-6-H)$, 7.16-7.15 (m, 2H, $\mathrm{CH}_{2}-\mathrm{Ph}-$ 4,5-H), 7.15-7.13 (m, 1H, C-Ph-6-H), $5.64(\mathrm{~s}, 1 \mathrm{H}$, $\mathrm{OH}), 5.25\left(\mathrm{~d}, 1 \mathrm{H}, J=14.6 \mathrm{~Hz}, \mathrm{Btz}-\mathrm{CH}_{2}\right), 4.97(\mathrm{~d}$, $\left.1 \mathrm{H}, J=14.6 \mathrm{~Hz}, \mathrm{Btz}-\mathrm{CH}_{2}\right), 3.62(\mathrm{~d}, 1 \mathrm{H}, J=13.7 \mathrm{~Hz}$, C-C $H_{2}$-Pip), 3.37 (d, $2 \mathrm{H}, J=21.6 \mathrm{~Hz}$, Pip- $\mathrm{CH}_{2}-\mathrm{Ph}$ ), $2.86\left(\mathrm{~d}, 1 \mathrm{H}, J=13.7 \mathrm{~Hz}, \mathrm{C}-\mathrm{CH}_{2}\right.$-Pip), 2.31-2.25 (m, 4H, Pip), 2.24-2.22 (m, 4H, Pip) ppm; ${ }^{13} \mathrm{C}$ NMR 
$\left(150 \mathrm{MHz}, \mathrm{CDCl}_{3}\right): \delta 145.57,139.53,131.53,130.84$, $130.28,128.52,127.11,126.99,124.40,123.67$, $119.56,115.69,114.04,111.34,73.83,62.05,60.71$, 54.78, 54.05, 52.89 ppm; MS $(m / z): 531.88[\mathrm{M}+\mathrm{H}]^{+}$.

2.4c 1-(1H-benzo[d][1,2,3]triazol-1-yl)-3-(4-(3,4-dichlorobenzyl)piperazin-1-yl)-2-(2,4-dichloro phenyl)propan-2-ol $(\boldsymbol{6 c})$ ): Yellow oil. Yield: $43.5 \%$. IR (KBr, $\left.\mathrm{cm}^{-1}\right)$ : 3441, 3112, 3029, 2986, 2866, 1609, 1587, $1504,1459,1344,1233,1141,977,876,786,684 \mathrm{~cm}^{-1}$. ${ }^{1} \mathrm{H} \mathrm{NMR}\left(600 \mathrm{MHz}, \mathrm{CDCl}_{3}\right): \delta 7.98(\mathrm{~d}, 1 \mathrm{H}, J=8.3 \mathrm{~Hz}$, Btz-4- $H), 7.73(\mathrm{~d}, 1 \mathrm{H}, J=8.6 \mathrm{~Hz}, \mathrm{Btz}-7-H), 7.71(\mathrm{~d}$, $1 \mathrm{H}, J=7.5 \mathrm{~Hz}, \mathrm{C}-\mathrm{Ph}-3-H), 7.47(\mathrm{t}, 1 \mathrm{H}, J=7.6 \mathrm{~Hz}$, $\left.\mathrm{CH}_{2}-\mathrm{Ph}-5-H\right), 7.42-7.35$ (m, 2H, Btz-5,6- $H$ ), 7.32-7.30 (m, 2H, C-Ph-5,6- $H$ ), 7.15 (d, $1 \mathrm{H}, J=8.6 \mathrm{~Hz}, \mathrm{CH}_{2}-\mathrm{Ph}-$ $5-H), 7.06\left(\mathrm{~d}, 1 \mathrm{H}, J=8.0 \mathrm{~Hz}, \mathrm{CH}_{2}-\mathrm{Ph}-6-H\right), 5.62$ (s, $1 \mathrm{H}, \mathrm{OH}), 5.26\left(\mathrm{~d}, 1 \mathrm{H}, J=14.6 \mathrm{~Hz}, \mathrm{Btz}-\mathrm{CH}_{2}\right), 4.97(\mathrm{~d}$, $\left.1 \mathrm{H}, J=14.6 \mathrm{~Hz}, \mathrm{Btz}-\mathrm{CH}_{2}\right), 3.63(\mathrm{~d}, 1 \mathrm{H}, J=13.7 \mathrm{~Hz}$, C-C $H_{2}$-Pip), 3.32 (d, $2 \mathrm{H}, J=21.6 \mathrm{~Hz}$, Pip-C $H_{2}$ ), 2.87 (d, $1 \mathrm{H}, J=13.7 \mathrm{~Hz}, \mathrm{Ph}-\mathrm{CH}_{2}$-Pip), 2.37-2.26 (m, 8H, Pip) ppm; ${ }^{13} \mathrm{C}$ NMR $\left(150 \mathrm{MHz}, \mathrm{CDCl}_{3}\right): \delta 145.78$, $139.95,135.08,134.89,134.57,133.27,130.89$, $130.49,130.06,129.25,128.67,127.55,126.88$, $123.58,123.65,119.59,111.39,73.70,60.74,58.22$, 54.70, 54.02, 52.83 ppm; MS $(m / z): 566.33[\mathrm{M}+\mathrm{H}]^{+}$.

2.4d 1-(1H-benzo[d][1,2,3]triazol-1-yl)-3-(4-(2-chlorobenzyl)piperazin-1-yl)-2-(2,4-dichloro phenyl)propan2-ol $(\boldsymbol{6} \boldsymbol{d})$ : Yellow oil. Yield: $42.5 \%$. IR $\left(\mathrm{KBr}, \mathrm{cm}^{-1}\right)$ : 3447, 3112, 3029, 2986, 2870, 1612, 1587, 1506, 1458, 1346, 1232, 1138, 982, 857, 783, $680 \mathrm{~cm}^{-1} ;{ }^{1} \mathrm{H}$ NMR $\left(600 \mathrm{MHz}, \mathrm{CDCl}_{3}\right): \delta: 7.99(\mathrm{~d}, 1 \mathrm{H}, J=8.3 \mathrm{~Hz}, \mathrm{Btz}-4-$ $H), 7.74(\mathrm{~d}, 1 \mathrm{H}, J=8.6 \mathrm{~Hz}, \mathrm{Btz}-7-H), 7.72(\mathrm{~d}, 1 \mathrm{H}, J=$ $7.5 \mathrm{~Hz}, \mathrm{C}-\mathrm{Ph} 3-H), 7.47-7.44\left(\mathrm{~m}, 1 \mathrm{H}, \mathrm{CH}_{2}-\mathrm{Ph}-3-H\right)$, 7.40-7.37 (m, 2H, Btz-5,6-H), 7.34-7.32 (m, 1H, CPh-5-H), 7.31-7.29 (m, $\left.1 \mathrm{H}, \mathrm{CH}_{2}-\mathrm{Ph}-4-H\right), 7.21-7.18$ (m, 1H, C-Ph-6- $H$ ), 7.16-7.15 (m, 2H, $\left.\mathrm{CH}_{2}-\mathrm{Ph}-5,6-H\right)$, $5.67(\mathrm{~s}, 1 \mathrm{H}, \mathrm{OH}), 5.27$ (d, $1 \mathrm{H}, J=14.6 \mathrm{~Hz}, \mathrm{Btz}-\mathrm{CH}_{2}$ ), $4.99\left(\mathrm{~d}, 1 \mathrm{H}, J=14.6 \mathrm{~Hz}, \mathrm{Btz}-\mathrm{CH}_{2}\right), 3.63(\mathrm{~d}, 1 \mathrm{H}, J=$ $13.7 \mathrm{~Hz}, \mathrm{C}-\mathrm{CH}_{2}$-Pip), 3.51 (d, $2 \mathrm{H}, J=21.6 \mathrm{~Hz}$, Pip$\left.\mathrm{CH}_{2}-\mathrm{Ph}\right), 2.88\left(\mathrm{~d}, 1 \mathrm{H}, \mathrm{J}=13.7 \mathrm{~Hz}, \mathrm{C}-\mathrm{CH}_{2}-\mathrm{Pip}\right), 2.32$ 2.27 (m, 8H, Pip) ppm; ${ }^{13} \mathrm{C}$ NMR $\left(150 \mathrm{MHz}, \mathrm{CDCl}_{3}\right)$ : $\delta 160.11,158.56,145.78,139.93,135.12,134.57$, $134.37,131.46,130.89,130.48,130.07,129.48$, $128.80,127.48,127.13,126.93,126.62,123.67$, $119.60,111.41,73.92,60.73,58.87,54.73,54.04$, $52.88 \mathrm{ppm} ; \mathrm{MS}(\mathrm{m} / \mathrm{z}): 531.88[\mathrm{M}+\mathrm{H}]^{+}$.

2.4e 1-(1H-benzo[d][1,2,3]triazol-1-yl)-2-(2,4-dichlorophenyl)-3-(4-(2-fluorobenzyl) piperazin-1-yl)propan2-ol (6e): Yellow oil. Yield: $44.0 \%$. IR $\left(\mathrm{KBr}, \mathrm{cm}^{-1}\right)$ : 3447, 3112, 3029, 2986, 2868, 1693, 1587, 1507, 1464, 1347, 1230, 1138, 959, 869, 785, $679 \mathrm{~cm}^{-1} ;{ }^{1} \mathrm{H}$ NMR $\left(600 \mathrm{MHz}, \mathrm{CDCl}_{3}\right): \delta: 7.98(\mathrm{~d}, 1 \mathrm{H}, J=8.3, \mathrm{~Hz}, \mathrm{Btz}-4-$ $H), 7.73(\mathrm{~d}, 1 \mathrm{H}, J=8.6 \mathrm{~Hz}, \mathrm{Btz}-7-H), 7.71(\mathrm{~d}, 1 \mathrm{H}, J=$ $7.5 \mathrm{~Hz}, \mathrm{C}-\mathrm{Ph}-3-H), 7.46-7.43\left(\mathrm{~m}, 1 \mathrm{H}, \mathrm{CH}_{2}-\mathrm{Ph}-3-H\right)$, 7.39-7.36 (m, 2H, Btz-5,6-H), 7.33-7.30 (m, 1H, CPh-5-H), 7.33-7.30 (m, 1H, $\left.\mathrm{CH}_{2}-\mathrm{Ph}-4-H\right), 7.21-7.18$ (m, 1H, C-Ph-6- $H$ ), 7.05 (t, $1 \mathrm{H}, J=7.4 \mathrm{~Hz}, \mathrm{CH}_{2}-\mathrm{Ph}-$ 6-H), 6.98 (t, $\left.1 \mathrm{H}, J=9.0 \mathrm{~Hz}, \mathrm{CH}_{2}-\mathrm{Ph}-5-H\right), 5.64$ (s, $1 \mathrm{H}, \mathrm{OH}), 5.24\left(\mathrm{~d}, 1 \mathrm{H}, J=14.6 \mathrm{~Hz}, \mathrm{Btz}-\mathrm{CH}_{2}\right), 4.97$ (d, $\left.1 \mathrm{H}, J=14.6 \mathrm{~Hz}, \mathrm{Btz}-\mathrm{CH}_{2}\right), 3.61(\mathrm{~d}, 1 \mathrm{H}, J=13.7 \mathrm{~Hz}$, C-C $H_{2}$-Pip), 3.48 (d, $2 \mathrm{H}, J=21.6 \mathrm{~Hz}$, Pip-C $\mathrm{H}_{2}-\mathrm{Ph}$ ), $2.85\left(\mathrm{~d}, 1 \mathrm{H}, J=13.7 \mathrm{~Hz}, \mathrm{C}-\mathrm{CH}_{2}\right.$-Pip), 2.30-2.27 (m, $8 \mathrm{H}$, Pip) ppm; ${ }^{13} \mathrm{C}$ NMR $\left(150 \mathrm{MHz}, \mathrm{CDCl}_{3}\right): \delta 162.25$, $145.80,139.98,135.14,134.57,131.48,130.88$, $130.48,130.07,128.80,127.51,127.11,126.93$, $123.88,123.65,119.59,115.32,111.41,73.90,60.75$, $54.92,54.76,54.06,52.59 \mathrm{ppm}$; MS $(\mathrm{m} / \mathrm{z}): 515.43$ $[\mathrm{M}+\mathrm{H}]^{+}$.

2.4f 1-(1H-benzo[d][1,2,3]triazol-1-yl)-3-(4-(2,4-dichlorobenzyl)piperazin-1-yl)-2-(2,4-dichloro phenyl)propan-2-ol (6f): Yellow oil. Yield: 45.5\%. IR (KBr, $\mathrm{cm}^{-1}$ ): 3440, 3112, 3029, 2986, 2854, 1610, 1587, $1507,1460,1350,1235,1141,983,872,782,682 \mathrm{~cm}^{-1}$. ${ }^{1} \mathrm{H}$ NMR $\left(600 \mathrm{MHz}, \mathrm{CDCl}_{3}\right): \delta: 7.99(\mathrm{~d}, 1 \mathrm{H}, J=$ $8.3 \mathrm{~Hz}, \mathrm{Btz}-4-H), 7.74$ (d, $1 \mathrm{H}, J=8.6 \mathrm{~Hz}, \mathrm{Btz}-7-H)$, 7.73-7.71 (m, 1H, C-Ph-3-H), 7.44-7.39 (m, 2H, Btz5,6-H), 7.34-7.31 (m, 2H, C-Ph-5,6-H), 7.16-7.13 $\left(\mathrm{m}, 2 \mathrm{H}, \mathrm{CH}_{2}-\mathrm{Ph}-5,6-H\right), 5.65(\mathrm{~s}, 1 \mathrm{H}, \mathrm{OH}), 5.27(\mathrm{~d}$, $\left.1 \mathrm{H}, J=14.6 \mathrm{~Hz}, \mathrm{Btz}-\mathrm{CH}_{2}\right), 4.97(\mathrm{~d}, 1 \mathrm{H}, J=14.6 \mathrm{~Hz}$, Btz-C $H_{2}$ ), 3.64 (d, $1 \mathrm{H}, J=13.7 \mathrm{~Hz}, \mathrm{C}-\mathrm{CH}_{2}$-Pip), 3.46 (d, $2 \mathrm{H}, J=21.6 \mathrm{~Hz}, \mathrm{Pip}-\mathrm{CH}_{2}-\mathrm{Ph}$ ), 2.87 (d, $1 \mathrm{H}, J=$ $13.7 \mathrm{~Hz}, \mathrm{C}-\mathrm{CH}_{2}$-Pip), 2.35-2.29 (m, 4H, Pip), 2.262.16 (s, 4H, Pip) ppm; ${ }^{13} \mathrm{C} \mathrm{NMR}\left(150 \mathrm{MHz}, \mathrm{CDCl}_{3}\right)$ : $\delta 145.78,139.93,135.01,134.84,134.59,133.32$, $131.46,130.90,130.48,130.06,129.24,128.80$, 127.54, 127.14, 126.93, 123.68, 119.60, 111.39, 73.93, $60.73,58.35,54.71,54.07,52.89 \mathrm{ppm} ; \mathrm{MS}(\mathrm{m} / \mathrm{z})$ : $566.33[\mathrm{M}+\mathrm{H}]^{+}$.

2.4g 1-(1H-benzo[d][1,2,3]triazol-1-yl)-2-(2,4-dichlorophenyl)-3-(4-(3-fluorobenzyl) piperazin-1-yl)propan2-ol $(\boldsymbol{6 g})$ : Yellow oil. Yield: $40.1 \%$. IR $\left(\mathrm{KBr}, \mathrm{cm}^{-1}\right)$ : 3447, 3112, 3029, 2986, 2868, 1697, 1611, 1589, 1505, $1458,1345,1235,1140,979,853,785,681 \mathrm{~cm}^{-1}$. ${ }^{1} \mathrm{H}$ NMR $\left(600 \mathrm{MHz}, \mathrm{CDCl}_{3}\right): \delta: 7.98(\mathrm{~d}, 1 \mathrm{H}, J=$ $8.3 \mathrm{~Hz}, \mathrm{Btz}-4-H), 7.73(\mathrm{~d}, 1 \mathrm{H}, J=8.6 \mathrm{~Hz}, \mathrm{Btz}-7-H)$, $7.71(\mathrm{~d}, 1 \mathrm{H}, J=7.5 \mathrm{~Hz}, \mathrm{C}-\mathrm{Ph}-3-H), 7.45-7.40(\mathrm{~m}$, 2H, Btz-5,6-H), 7.33-7.31 (m, 1H, C-Ph-5-H), 7.25 (s, $\left.1 \mathrm{H}, \mathrm{CH}_{2}-\mathrm{Ph}-5-H\right), 7.15-7.13$ (m, $\left.1 \mathrm{H}, \mathrm{C}-\mathrm{Ph}-6-H\right)$, 6.95-6.99 (m, 2H, $\left.\mathrm{CH}_{2}-\mathrm{Ph}-4,6-H\right), 6.91-6.88(\mathrm{~m}, 1 \mathrm{H}$, $\left.\mathrm{CH}_{2}-\mathrm{Ph}-2-H\right), 5.65(\mathrm{~s}, 1 \mathrm{H}, \mathrm{OH}), 5.25(\mathrm{~d}, 1 \mathrm{H}, J=$ $\left.14.6 \mathrm{~Hz}, \mathrm{Btz}-\mathrm{CH}_{2}\right), 4.98(\mathrm{~d}, 1 \mathrm{H}, J=14.6 \mathrm{~Hz}, \mathrm{Btz}-$ $\left.\mathrm{CH}_{2}\right), 3.63\left(\mathrm{~d}, 1 \mathrm{H}, J=13.7 \mathrm{~Hz}, \mathrm{C}-\mathrm{CH}_{2}\right.$-Pip), 3.37 
$\left(\mathrm{d}, 2 \mathrm{H}, J=21.6 \mathrm{~Hz}\right.$, Pip-C $\left.H_{2}-\mathrm{Ph}\right), 2.86(\mathrm{~d}, 1 \mathrm{H}, J$ $\left.=13.7 \mathrm{~Hz}, \mathrm{C}-\mathrm{CH}_{2}-\mathrm{Pip}\right), 2.31-2.31$ (m, 4H, Pip), 2.25-2.24 (m, 4H, pip) Ppm; ${ }^{13} \mathrm{C}$ NMR $(150 \mathrm{MHz}$, $\left.\mathrm{CDCl}_{3}\right): \delta 162.52,145.77,139.95,135.11,134.56$, $131.46,130.89,130.48,128.79,127.50,127.11$, $126.92,124.40,119.60,114.07,111.38,73.87,62.07$, 60.71, 54.77, 54.05, $52.91 \mathrm{ppm}$; MS $(\mathrm{m} / \mathrm{z}): 515.43$ $[\mathrm{M}+\mathrm{H}]^{+}$.

2.4h 1-(1H-benzo[d][1,2,3]triazol-1-yl)-3-(4-(4-chlorobenzyl)piperazin-1-yl)-2-(2,4-dichloro phenyl)propan2-ol $(\boldsymbol{6} \boldsymbol{h})$ : Yellow oil. Yield: $41.3 \%$. IR $\left(\mathrm{KBr}, \mathrm{cm}^{-1}\right)$ : 3447, 3112, 3029, 2986, 2870, 1701, 1660, 1612, 1589, 1509, 1464, 1353, 1236, 1137, 973, 866, 785, $680 \mathrm{~cm}^{-1} .{ }^{1} \mathrm{H}$ NMR $\left(600 \mathrm{MHz}, \mathrm{CDCl}_{3}\right): \delta: 7.98(\mathrm{~d}, 1 \mathrm{H}$, $J=8.3 \mathrm{~Hz}, \mathrm{Btz}-4-H), 7.73(\mathrm{~d}, 1 \mathrm{H}, J=8.6 \mathrm{~Hz}, \mathrm{Btz}-$ $7-H), 7.71(\mathrm{~d}, 1 \mathrm{H}, J=7.5 \mathrm{~Hz}, \mathrm{C}-\mathrm{Ph}-3-H), 7.45-7.40$ (m, 2H, Btz-5,6-H), 7.33-7.31 (m, 1H, C-Ph-5-H), 7.24-7.22 (m, 2H, CH $-\mathrm{Ph}-3,5-H), 7.17(\mathrm{~s}, 1 \mathrm{H}, \mathrm{C}-$ Ph-6- $H$ ), 7.16-7.14 (m, 2H, $\left.\mathrm{CH}_{2}-\mathrm{Ph}-2,6-H\right), 5.61$ (s, $1 \mathrm{H}, \mathrm{OH}), 5.26\left(\mathrm{~d}, 1 \mathrm{H}, J=14.6 \mathrm{~Hz}, \mathrm{Btz}-\mathrm{CH}_{2}\right), 4.97(\mathrm{~d}$, $\left.1 \mathrm{H}, J=14.6 \mathrm{~Hz}, \mathrm{Btz}-\mathrm{C} H_{2}\right), 3.63(\mathrm{~d}, 1 \mathrm{H}, J=13.7 \mathrm{~Hz}$, C-C $H_{2}$-Pip), 3.36 (d, $2 \mathrm{H}, J=21.6 \mathrm{~Hz}, \mathrm{Pip}-\mathrm{CH}_{2}-\mathrm{Ph}$ ), $2.86\left(\mathrm{~d}, 1 \mathrm{H}, J=13.7 \mathrm{~Hz}, \mathrm{C}-\mathrm{CH}_{2}\right.$-Pip), $2.31-2.25$ (m, 4H, Pip), 2.25-2.17 (m, 4H, Pip) ppm; ${ }^{13} \mathrm{C}$ NMR $\left(150 \mathrm{MHz}, \mathrm{CDCl}_{3}\right): \delta 145.78,139.90,134.61$, $133.05,131.47,130.91,130.35,130.05,128.46$, $127.53,127.14,123.68,119.63,111.35,73.95,61.90$, $60.69,54.73,53.93,52.83 \mathrm{ppm}$; MS $(\mathrm{m} / \mathrm{z}): 531.88$ $[\mathrm{M}+\mathrm{H}]^{+}$.

2.4i 1-(1H-benzo[d][1,2,3]triazol-1-yl)-2-(2,4-dichlorophenyl)-3-(4-(4-fluorobenzyl) piperazin-1-yl)propan2-ol (6i): Yellow oil. Yield: $44.8 \%$. IR $\left(\mathrm{KBr}, \mathrm{cm}^{-1}\right)$ : 3447, 3112, 3029, 2986, 2872, 1698, 1609, 1589, 1508, $1456,1347,1235,1135,975,870,783,680 \mathrm{~cm}^{-1}$. ${ }^{1} \mathrm{H}$ NMR $\left(600 \mathrm{MHz}, \mathrm{CDCl}_{3}\right): \delta: 7.98(\mathrm{~d}, 1 \mathrm{H}, J=$ $8.3 \mathrm{~Hz}, \mathrm{Btz}-4-H), 7.73(\mathrm{~d}, 1 \mathrm{H}, J=8.6 \mathrm{~Hz}, \mathrm{Btz}-7-$ $H), 7.71(\mathrm{~d}, 1 \mathrm{H}, J=7.5 \mathrm{~Hz}, \mathrm{C}-\mathrm{Ph}-3-H), 7.45-7.40$ $(\mathrm{m}, 2 \mathrm{H}$, Btz-5,6-H), 7.34-7.31 (m, 1H, C-Ph-5$H$ ), 7.19-7.17 (t, 2H, $\left.J=8.6 \mathrm{~Hz}, \mathrm{CH}_{2}-\mathrm{Ph} 2,6-H\right)$, 7.15-7.14 (s, 1H, C-Ph-6- $H$ ), 6.94 (t, 2H, $J=8.6 \mathrm{~Hz}$, $\left.\mathrm{CH}_{2}-\mathrm{Ph}-3,5-H\right), 5.62(\mathrm{~s}, 1 \mathrm{H}, \mathrm{OH}), 5.27(\mathrm{~d}, 1 \mathrm{H}, J=$ $\left.14.6 \mathrm{~Hz}, \mathrm{Btz}-\mathrm{CH}_{2}\right), 4.97$ (d, $1 \mathrm{H}, J=14.6 \mathrm{~Hz}, \mathrm{Btz}-$ $\mathrm{CH}_{2}$ ), $3.61\left(\mathrm{~d}, 1 \mathrm{H}, \mathrm{J}=13.7 \mathrm{~Hz}, \mathrm{C}-\mathrm{CH}_{2}\right.$-Pip), 3.35 $\left(\mathrm{d}, 2 \mathrm{H}, J=21.6 \mathrm{~Hz}\right.$, Pip-C $\left.\mathrm{H}_{2}-\mathrm{Ph}\right), 2.86(\mathrm{~d}, 1 \mathrm{H}, J=$ $13.7 \mathrm{~Hz}, \mathrm{C}-\mathrm{CH}_{2}$-Pip), 2.31-2.23 (m, 8H, Pip) ppm; ${ }^{13} \mathrm{C}$ NMR $\left(150 \mathrm{MHz}, \mathrm{CDCl}_{3}\right): \delta 162.98,145.77$, $139.88,135.15,134.60,134.56,131.46,130.90$, $130.49,130.04,128.80,127.52,127.14,126.93$, $123.67,119.62,115.17,115.03,111.34,73.95,61.92$, 60.71, 54.69, 54.30, $52.76 \mathrm{ppm}$; MS $(\mathrm{m} / \mathrm{z}): 515.43$ $[\mathrm{M}+\mathrm{H}]^{+}$.

\subsection{Biological assay procedures}

Minimal inhibitory concentration (MIC, $\mu \mathrm{g} / \mathrm{mL}$ ) is defined as the lowest concentration of the new compounds that completely inhibited the growth of bacteria, by means of standard two-fold serial dilution method in 96-well micro-test plates according to the National Committee for Clinical Laboratory Standards (NCCLS). ${ }^{3}$ The tested microorganism strains were provided by the School of Pharmaceutical Sciences, Southwest University and the College of Pharmacy, Third Military Medical University. Chloromycin, Norfloxacin and Fluconazole were used as control drugs. To ensure that the solvent had no effect on bacterial growth, a control test was performed with test medium supplemented with DMSO at the same dilutions as used in the experiment. All the bacteria and fungi growth was monitored visually and spectrophotometrically. The lowest concentration (highest dilution) required to arrest the growth of bacteria was regarded as minimal inhibitory concentration (MIC).

2.5a Antibacterial assays: The prepared compounds 3-6 were evaluated for their antibacterial activities against Methicillinresistant staphylococcus aureus N315, Staphylococcus aureus ATCC25923, Bacillus subtilis ATCC6633 and Micrococcus luteus ATCC 4698 as Gram-positive, Escherichia coli DH52, Bacillus proteus ATCC13315, Shigella dysenteriae ATCC51252 and Pseudomonas aeruginosa ATCC27853 as Gramnegative bacteria. The bacterial suspension was adjusted with sterile saline to a concentration of $1 \times$ $10^{5} \mathrm{CFU} / \mathrm{mL}$. The compounds were dissolved in DMSO to prepare the stock solutions. The compounds and reference drugs were prepared in Mueller-Hinton broth (Guangdong huaikai microbial sci.\& tech co., Ltd, Guangzhou, Guangdong, China) by two-fold serial dilution to obtain the required concentrations of 512 , $256,128,64,32,16,8,4,2,1,0.5 \mu \mathrm{g} / \mathrm{mL}$. These dilutions were inoculated and incubated at $37^{\circ} \mathrm{C}$ for $24 \mathrm{~h}$. To ensure that the solvent had no effect on bacterial growth, a control test was performed with test medium supplemented with DMSO at the same dilutions as used in the experiment.

2.5b Antifungal assays: The synthesized compounds 3-6 were also evaluated for their antifungal activities against Candida albicans, Candida utilis, Candida mycoderma and Beer yeast. A spore suspension in sterile distilled water was prepared from 1-day old culture of the fungi growing on Sabouraud agar (SA) media. The final spore concentration was $1-5 \times 10^{3}$ spore $/ \mathrm{mL}$. From the stock solutions of the 
tested compounds and reference antifungal fluconazole, dilutions in sterile RPMI 1640 medium (Neuronbc Laboraton Technology CO., Ltd, Beijing, China) were made resulting in eleven wanted concentrations $(0.5-$ $512 \mu \mathrm{g} / \mathrm{mL}$ ) of each tested compounds. These dilutions were inoculated and incubated at $35^{\circ} \mathrm{C}$ for $24 \mathrm{~h}$. The MIC of drug was defined as the first well with an approximate $80 \%$ reduction in growth compared to the growth of the drug-free well.

\section{Results and Discussion}

\subsection{Chemistry}

The synthetic route of target compounds is outlined in scheme 1 . The desired benzotriazole piperazine hybrids were prepared via multistep reactions from commercially available substituted benzene, benzotriazole and piperazine. The intermediate $\mathbf{2}$ could be efficiently prepared in satisfactory yield $(90 \%)$ by acetylation of 1,3dichlorobenzene by chloroacetyl chloride, and then Nalkylation reaction was performed to afford the benzotriazolyl ethanone $\mathbf{3}$ in good yield (77\%). Further epoxidation of compound $\mathbf{3}$ in toluene by trimethyl sulfoxonium iodide (TMSI) and 20\% sodium hydroxide at $60^{\circ} \mathrm{C}$ produced the benzotriazolyl oxirane 4 in $68.2 \%$ yield. The intermediates $\mathbf{5 a - i}$ were prepared by the $\mathrm{N}$-alkylation of piperazine by different halobenzyls in good yields (60-65\%). The target compounds $\mathbf{6 a - i}$ were afforded by the reaction of compound $\mathbf{5 a - i}$ with compound 4 in ethanol using sodium bicarbonate as base at $70^{\circ} \mathrm{C}$. All new compounds were characterized by ${ }^{1} \mathrm{H}$ NMR, ${ }^{13} \mathrm{C}$ NMR, IR and MS spectra.

\subsection{Antimicrobial activities}

The antimicrobial results, depicted in table 1 , revealed that benzotriazolyl ethanone $\mathbf{3}$ and halobenzyl substituted

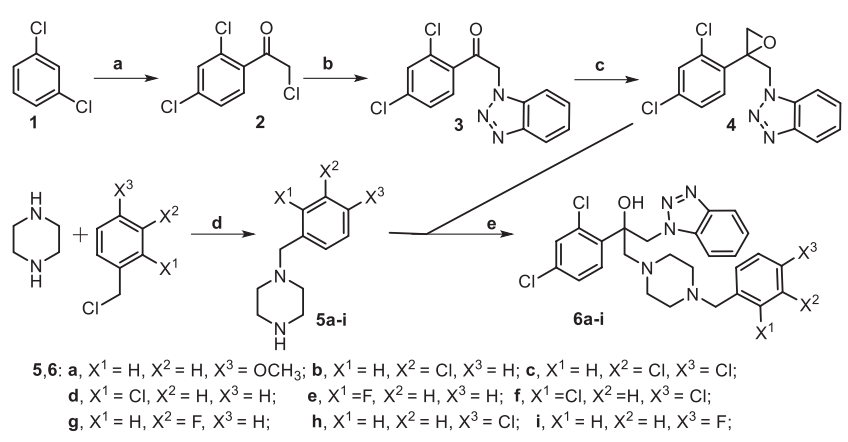

Scheme 1. Reagents and conditions: a) $\mathrm{ClCOCH}_{2} \mathrm{Cl}$, $\mathrm{AlCl}_{3}, \mathrm{CH}_{2} \mathrm{Cl}_{2}, \mathrm{rt}, 2-3 \mathrm{~h}$; b) 1H-benzotriazole, $\mathrm{K}_{2} \mathrm{CO}_{3}$, $\mathrm{CH}_{3} \mathrm{CN}, 80^{\circ} \mathrm{C}, 8 \mathrm{~h}$; c) TMSI, $20 \% \mathrm{NaOH}$, toluene, $6^{\circ} \mathrm{C}, 8 \mathrm{~h}$; d) $\mathrm{CH}_{3} \mathrm{CN}$, rt, 0.5-1 h; e) $\mathrm{NaHCO}_{3}$, EtOH, $70^{\circ} \mathrm{C}, 18-24 \mathrm{~h}$; $\mathrm{HCOOH}, \mathrm{rt}$. piperazines 5a-i showed poor inhibition against most of the tested bacterial strains. In comparison with benzotriazolyl ethanone $\mathbf{3}$, benzotriazolyl oxirane $\mathbf{4}$ displayed stronger antibacterial activity and broader antibacterial spectrum. Benzotriazolyl oxirane $\mathbf{4}$ gave good inhibition against $B$. subtilis (MIC $=16 \mu \mathrm{g} / \mathrm{mL}$ ), which was two-fold active of clinical drug Chloromycin (MIC $=32 \mu \mathrm{g} / \mathrm{mL}$ ). These results suggested that the substitution of carbonyl group into anoxirane moiety might be beneficial to the antibacterial activities. For the tested benzotriazole piperazines $\mathbf{6 a}-\mathbf{i}$, all of them displayed good inhibitory efficacy toward both Grampositive and Gram-negative bacteria with MIC values in the range of $8-128 \mu \mathrm{g} / \mathrm{mL}$, which were more active than their precursors $\mathbf{4}$ and $\mathbf{5 a}-\mathbf{i}$. Among the target molecules, compound 6a with 4-methoxyl benzyl group exhibited moderate antibacterial activities against the tested strains with MIC values ranging from 64 to $128 \mu \mathrm{g} / \mathrm{mL}$. The replacement of 4-methoxyl group by 3,4-dichlorobenzyl, 2,4-dichlorobenzyl and 4-chlorobenzyl fragments to afford compounds $\mathbf{6 c}, \mathbf{6 f}$ and $\mathbf{6 h}$, respectively, that showed comparable activities to reference drug chloromycin against the tested bacterial strains with the MIC values in the range of 4-32 $\mu \mathrm{g} / \mathrm{mL}$. Notably, compound 6f exhibited good inhibition against MRSA (MIC $=4 \mu \mathrm{g} / \mathrm{mL})$, which was equivalent or even better than the reference drugs chloromycin (MIC $=8 \mu \mathrm{g} / \mathrm{mL}$ ) and norfloxacin $(\mathrm{MIC}=4 \mu \mathrm{g} / \mathrm{mL})$.

The antifungal evaluation in vitro showed that most of these compounds were more sensitive against fungal strains than bacterial strains. The antifungal activity of benzotriazolyl intermediate $\mathbf{4}$ against Beer yeast ATCC 9763 was equivalent to reference drug fluconazole $(\mathrm{MIC}=16 \mu \mathrm{g} / \mathrm{mL}$ ). The target compounds $\mathbf{6 a}-\mathbf{i}$ with moderate to good antifungal activities against the tested strains. Especially, the 2,4dichlorobenzyl compound $\mathbf{6 f}$ displayed strong antifungal activity against Beer yeast ATCC9763 with MIC value of $4 \mu \mathrm{g} / \mathrm{mL}$, which was 3 -fold more active than fluconazole. This compound also showed comparable inhibition with fluconazole against Candida utilis ATCC9950 and Candida mycoderma (MIC $=4$ and $8 \mu \mathrm{g} / \mathrm{mL}$, respectively). This showed that this compound is worthy of further investigation as a antifungal agent.

These bioactive evaluation results indicated that the combination of benzotriazole and piperazine through tertiaryalcohol frame could effectively improve the antimicrobial activity, which should be attributed to the substituted halobenzyl groups on piperazine to some extent. 
Table 1. Antimicrobial activities for the prepared compounds expressed as MIC $(\mu \mathrm{g} / \mathrm{mL})^{\mathrm{a}, \mathrm{b}, \mathrm{c}}$.

\begin{tabular}{|c|c|c|c|c|c|c|c|c|c|c|c|c|}
\hline \multirow[b]{2}{*}{ Compds } & \multicolumn{4}{|c|}{ Gram-positive bacteria } & \multicolumn{4}{|c|}{ Gram-negative bacteria } & \multicolumn{4}{|c|}{ Fungi } \\
\hline & $M R S A$ & $S . A$ & $B . S$ & $M . L$ & E.C & B.P & $S . D$ & $P . A$ & C.A & C.U & C.M & B.Y \\
\hline 3 & 512 & 512 & 512 & 512 & 32 & 512 & 512 & 64 & 512 & 512 & 512 & 512 \\
\hline 4 & 256 & 256 & 16 & 32 & 128 & 256 & 256 & 256 & 128 & 32 & 64 & 16 \\
\hline $5 a$ & 512 & 512 & 256 & 64 & 512 & 256 & 512 & 512 & 256 & 256 & 256 & 128 \\
\hline $5 b$ & 64 & 256 & 128 & 128 & 128 & 128 & 128 & 128 & 32 & 64 & 32 & 64 \\
\hline $5 c$ & 128 & 128 & 128 & 64 & 128 & 64 & 256 & 128 & 64 & 128 & 64 & 128 \\
\hline $5 d$ & 128 & 512 & 256 & 256 & 128 & 64 & 128 & 64 & 64 & 16 & 128 & 128 \\
\hline $5 e$ & 128 & 128 & 64 & 128 & 256 & 128 & 256 & 256 & 128 & 64 & 64 & 32 \\
\hline $5 f$ & 256 & 256 & 128 & 256 & 256 & 128 & 256 & 256 & 128 & 128 & 64 & 256 \\
\hline $5 \mathrm{~g}$ & 256 & 256 & 128 & 256 & 256 & 256 & 256 & 128 & 64 & 256 & 128 & 256 \\
\hline $5 \mathrm{~h}$ & 128 & 128 & 256 & 256 & 512 & 128 & 128 & 512 & 128 & 128 & 128 & 128 \\
\hline $5 i$ & 64 & 128 & 256 & 256 & 128 & 256 & 256 & 128 & 64 & 128 & 128 & 256 \\
\hline $6 \mathbf{a}$ & 128 & 64 & 128 & 64 & 64 & 64 & 64 & 64 & 64 & 128 & 64 & 64 \\
\hline $6 \mathrm{~b}$ & 32 & 32 & 16 & 32 & 16 & 32 & 32 & 32 & 32 & 16 & 16 & 32 \\
\hline 6c & 8 & 32 & 16 & 32 & 16 & 32 & 16 & 16 & 16 & 16 & 16 & 16 \\
\hline $6 d$ & 32 & 16 & 32 & 32 & 32 & 32 & 16 & 32 & 32 & 16 & 16 & 32 \\
\hline $6 \mathrm{e}$ & 64 & 32 & 32 & 32 & 16 & 16 & 16 & 32 & 64 & 32 & 64 & 64 \\
\hline $6 f$ & 4 & 16 & 16 & 8 & 16 & 16 & 16 & 16 & 4 & 4 & 8 & 4 \\
\hline $6 \mathrm{~g}$ & 64 & 64 & 32 & 64 & 32 & 32 & 64 & 64 & 16 & 32 & 16 & 64 \\
\hline $6 \mathrm{~h}$ & 32 & 32 & 16 & 32 & 32 & 16 & 32 & 32 & 32 & 32 & 16 & 32 \\
\hline $6 \mathbf{i}$ & 32 & 32 & 64 & 32 & 32 & 16 & 32 & 32 & 16 & 16 & 16 & 16 \\
\hline $\mathbf{A}$ & 8 & 16 & 32 & 8 & 32 & 16 & 16 & 32 & - & - & - & - \\
\hline B & 4 & 1 & 2 & 1 & 4 & 4 & 1 & 2 & - & - & - & - \\
\hline C & - & - & - & - & - & - & - & - & 1 & 4 & 8 & 16 \\
\hline
\end{tabular}

${ }^{a}$ Minimum inhibitory concentrations were determined by micro broth dilution method for microdilution plates.

${ }^{\mathrm{b}}$ MRSA, Methicillin resistant staphylococcus aureus N315; S.A, Staphylococcus aureus ATCC25923; B.S, Bacillus subtilis ATCC6633; M.L, Micrococcus luteus ATCC 4698; E.C, Escherichia coli DH52; B.P, Bacillus proteus ATCC13315; S.D, Shigella dysenteriae ATCC51252; P.A, Pseudomonas aeruginosa ATCC2785. C.A, Candida albicans ATCC90029; C.U, Candida utilis ATCC9950; C.M, Candida mycoderma; B.Y, Beer yeast ATCC9763.

${ }^{\mathrm{c}} \mathbf{A}=$ Chloromycin; $\mathbf{B}=$ Norfloxacin; $\mathbf{C}=$ Fluconazole.

\subsection{Interaction with calf thymus DNA}

3.3a Absorption spectra of DNA in the presence of compound $6 f$ : In recent decades, DNA as an important target for organic molecules has attracted increasing attention in biomedical chemistry. The investigation of interactions between small molecules with DNA has become convenient and helpful for the rational design and construction of novel and efficient drugs targeted to DNA. Calf thymus DNA is commonly selected as DNA model due to its medical importance, low cost as well as ready availability. Therefore, with the aim to explore the possible mechanism of antimicrobial action, the binding studies between the highly active compound 6f and calf thymus DNA were carried out by UV-Vis spectroscopic method.

In UV-Vis absorption spectroscopy, hyperchromism and hypochromism are important spectral features to distinguish the change of DNA double-helical structure, which arise from the breakage of the DNA duplex secondary structure as well as the stabilization of the DNA duplex by either intercalation binding mode or the electrostatic effect of small molecules. ${ }^{36}$ The observed large hypochromism might indicate a close proximity of the aromatic chromophore to the DNA bases due to the strong interaction between the electronic states of intercalating chromophore and that of the DNA base pairs. ${ }^{37}$ With a fixed concentration of DNA, UV-Vis absorption spectra were recorded with the increasing amount of compound 6f. As shown in figure 3, UV-Vis spectra displayed that the maximum absorption peak of DNA at $255 \mathrm{~nm}$ exhibited proportional increase with increasing concentration of $6 \mathbf{6}$. Meanwhile, the absorbance of simple sum of free DNA and free compound $\mathbf{6 f}$ was slightly greater than the measured value for $6 \mathbf{f}$-DNA complex. This suggested that a weak hypochromic effect existed between DNA and compound $\mathbf{6 f}$. Furthermore, the intercalation of the aromatic chromophore of compound $\mathbf{6 f}$ into the helix and the strong overlap of $\pi-\pi *$ states of the large $\pi$-conjugated system with the electronic states of DNA bases were consistent with the observed spectral changes. 


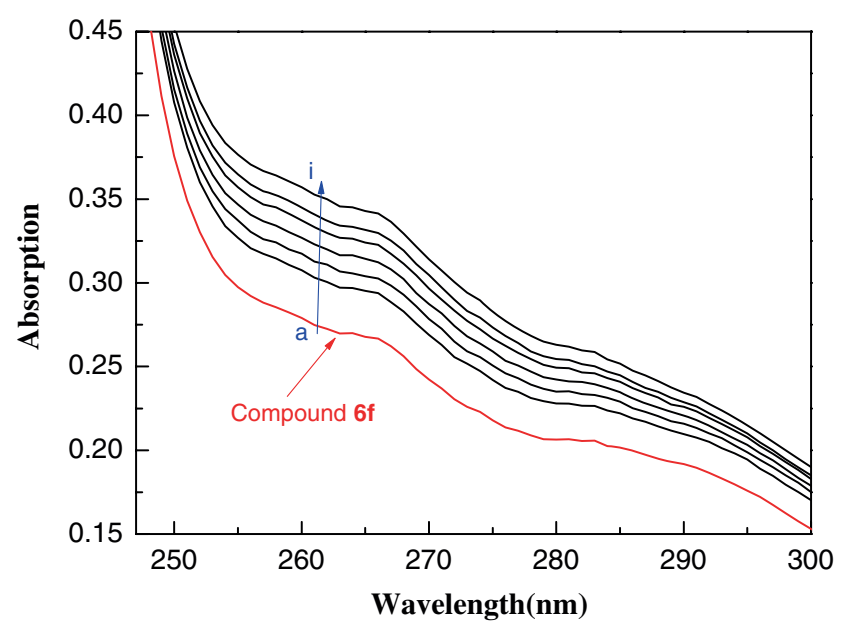

Figure 3. UV absorption spectra of compound $\mathbf{6} f$ with different concentrations of DNA $(\mathrm{pH}=7.4, \mathrm{~T}=296 \mathrm{~K})$. $c($ compound $\mathbf{6 f})=4.0 \times 10^{-5} \mathrm{~mol} / \mathrm{L}$, and $c(\mathrm{DNA})=0-$ $9.00 \times 10^{-6} \mathrm{~mol} / \mathrm{L}$ for curves $a-g$ respectively at increment $0.3 \times 10^{-5} \mathrm{~mol} / \mathrm{L}$.

Based on the variations in the absorption spectra (figure 3), equation (1) ${ }^{38}$ was used to calculate the intrinsic binding constant $(\mathrm{K})$.

$$
\frac{1}{\left|\xi_{\mathrm{a}}-\xi_{\mathrm{F}}\right|}=\frac{1}{\left|\xi_{\mathrm{b}}-\xi_{\mathrm{F}}\right|}+\frac{1}{\left|\xi_{\mathrm{b}}-\xi_{\mathrm{F}}\right| D K}
$$

Here, D is the concentration of DNA in base pairs, $\xi_{a}, \xi_{b}$, and $\xi_{F}$ correspond to the apparent absorption coefficient, $\left(\mathrm{A}_{\text {obsvd }} / \mathrm{D}\right)$ of the compound in the presence of DNA, extinction coefficient of the free compound $\left(6.98 \times 10^{3} \mathrm{~L} / \mathrm{mol} \mathrm{cm}^{-1}\right)$, and the extinction coefficient of the compound when fully bound to DNA $(10.24 \times$ $\left.10^{3} \mathrm{~L} / \mathrm{molcm}^{-1}\right)$, respectively. In the plot of $1 /\left|\xi_{a}-\xi_{F}\right|$ versus 1/D (figure S21 in Supplementary Information), equilibrium binding constant $(\mathrm{K})$ is given by the ratio of intercept to slope $\left(\mathrm{K}=2.2 \times 10^{4} \mathrm{~L} / \mathrm{mol}\right)$.

\section{3b Absorption spectra of NR interaction with DNA:} The absorption spectra of competitive interaction of compound $\mathbf{6 f}$ were investigated to further understand the interaction between compound $\mathbf{6} \mathbf{f}$ and DNA. Neutral Red (NR) is a planar phenazine dye and is structurally similar with other planar dyes, which possesses lower toxicity, higher stability and more convenient application in comparison to other common probes. In recent years, the binding of NR and DNA in intercalation mode has been sufficiently demonstrated by spectrophotometric and electrochemical techniques. ${ }^{39}$ Therefore, NR was employed as a spectral probe to investigate the binding mode of compound $\mathbf{6 f}$ with DNA in present work. The absorption spectra of the NR dye upon the addition of DNA were showed in figure 4. It is apparent that the absorption peak of the NR

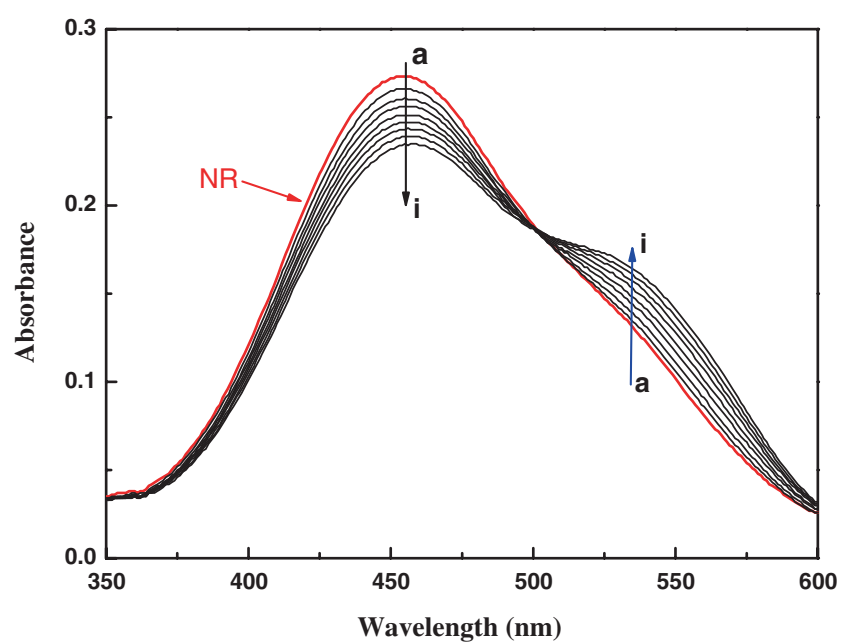

Figure 4. UV absorption spectra of NR in the presence of DNA at $\mathrm{pH} 7.4$ and room temperature. $c(\mathrm{NR})=2 \times$ $10^{-5} \mathrm{~mol} / \mathrm{L}$, and $c(\mathrm{DNA})=0-3.81 \times 10^{-5} \mathrm{~mol} / \mathrm{L}$ for curves $a-I$, respectively, at increment $0.47 \times 10^{-5} \mathrm{~mol} / \mathrm{L}$.

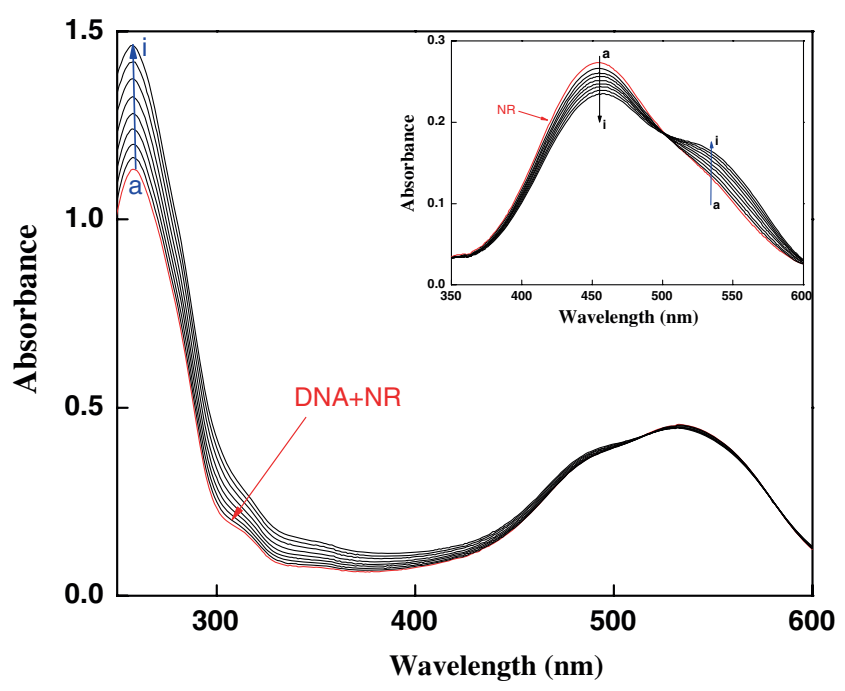

Figure 5. Absorption spectra of the competitive reaction between compound $\mathbf{6} \mathbf{f}$ and neutral red with DNA. $c$ (DNA) $=4.23 \times 10^{-5} \mathrm{~mol} / \mathrm{L}, c(\mathrm{NR})=2 \times 10^{-5} \mathrm{~mol} / \mathrm{L}$, and $c($ compound $\mathbf{6 f})=0-2.5 \times 10^{-5} \mathrm{~mol} / \mathrm{L}$ for curves $a-I$, respectively, at increment $0.20 \times 10^{-5} \mathrm{~mol} / \mathrm{L}$. (Inset) Absorption spectra of the system of competitive reaction between compound $6 \mathbf{f}$ and NR with DNA with the increasing concentration of 6f in the wavelength range of 350-600 nm.

at around $460 \mathrm{~nm}$ showed gradual decrease with the increasing concentration of DNA, and a new band at around $530 \mathrm{~nm}$ developed. This was attributed to the formation of the new DNA-NR complex. An isosbestic point at $504 \mathrm{~nm}$ provided evidence of DNA-NR complex formation.

\section{3c Absorption spectra of competitive interaction of} compound $6 f$ and NR with DNA: Figure 5 displayed the absorption spectra of the competitive binding between NR and 6f with DNA. As shown, with the 


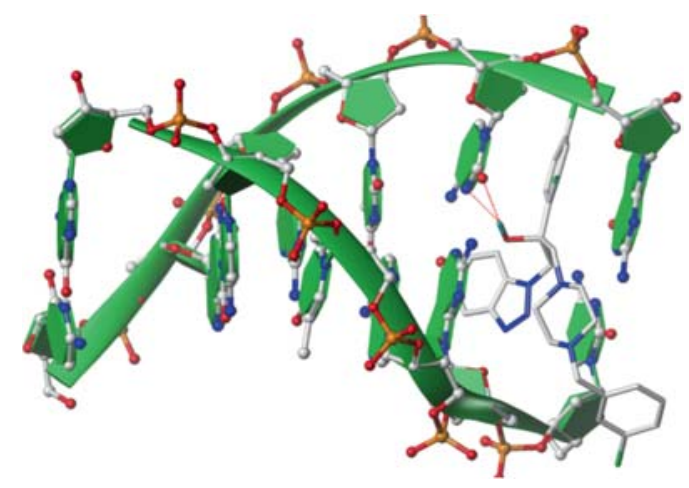

Figure 6. Molecular modeling of compound $6 \mathbf{f}$ and DNA hexamer duplex (PDB: 3FT6). The dashed lines represent the hydrogen bonding interactions between compound $\mathbf{6} \mathbf{f}$ and DNA hexamer duplex (Total binding score is 4.27).

gradually increasing concentration of compound $\mathbf{6 f}$, the absorption around $530 \mathrm{~nm}$ of the DNA-NR complex decreased, however, a slight intensity increase was observed in the developing band around $516 \mathrm{~nm}$. Compared with the absorption band at around $516 \mathrm{~nm}$ of NR-DNA complex (figure 5), the absorbance at the same wavelength in figure 5 (inset) exhibited the reverse process. The results suggested that compound $6 \mathbf{f}$ intercalated into the double helix of DNA by substituting for NR in the DNA-NR complex.

\subsection{Molecular docking of compound $\mathbf{6} f$ and DNA hexamer duplex}

DNA is a good target for antimicrobial agents due to its base pairs with rich electrons. ${ }^{40,41}$ Generally, bioactive molecules could bind to DNA in various modes such as electrostatic, groove and intercalative binding. Among them, the intercalative mode is the most important one, in which molecules could intercalate between the base pairs of double helix DNA, then greatly affect or even damage DNA. ${ }^{42}$

Molecular docking study is a useful method to investigate the binding modes of small molecules to DNA. ${ }^{43}$ So far, the full-length 3D structure of calf thymus DNA is not available in Protein Data Bank (PDB). Since calf thymus DNA is B-DNA, we chose the CT-DNA sequence d(CGATCG) $)_{2}$ (PDB code: 3FT6) as receptor model ${ }^{44,45}$ In this work, molecular docking study was performed between compound $\mathbf{6 f}$ and DNA hexamer duplex to understand the binding model. The docking mode with the lowest binding free energy $\left(-24.33 \mathrm{~kJ} \cdot \mathrm{mol}^{-1}\right)$ is shown in figures 6 and 7 . The results indicated that compound $\mathbf{6 f}$ intercalated into the duplexes of DNA, and the hydrogen atom in the hydroxyl group of compound $6 \mathbf{f}$ formed hydrogen bonds with the guanine of DNA, thus preventing the

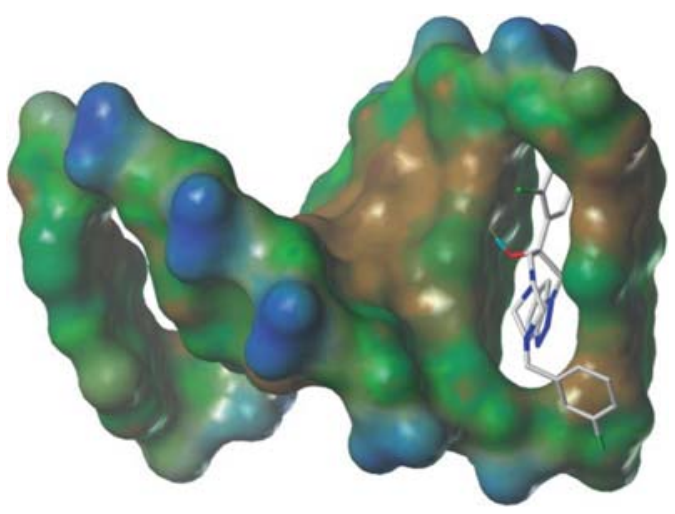

Figure 7. Stereoview of the conformation of compound $\mathbf{6 f}$ intercalated to DNA hexamer duplex to form compound $\mathbf{6 f}-$ DNA complex.

formation of hydrogen bond between cytosine in DNA. This kind of interaction resulted in decreased stability of DNA, thus inhibiting its physiological function. All these suggested that the simulation results agree well with the above spectral experiment results.

\section{Conclusions}

In conclusion, novel type of benzotriazoles were successfully synthesized for the first time via an easy, convenient and efficient synthetic procedure. The antimicrobial tests demonstrated that most of the new compounds exhibited moderate to good antibacterial and antifungal activity against the tested strains. In particular, compound $6 \mathbf{f}$ bearing 2,4-dichlorobenzyl fragment exhibited superior bioactivities compared to other analogues with MIC values ranging from 4 to $16 \mu \mathrm{g} / \mathrm{mL}$. The specific interaction of compound $\mathbf{6 f}$ with calf thymus DNA showed that this compound could intercalate into DNA to form compound $\mathbf{6 f - D N A}$ complex, which might further block DNA replication to explain its good antimicrobial activity. Molecular docking studies suggested that compound 6f projected into basepairs of DNA hexamer duplex forming two hydrogen bonds with guanine of DNA. All these results indicate a promising starting point to optimize the structures of benzotriazole-derived tertiary alcohols as potent antimicrobial agents.

\section{Supplementary Information}

The spectroscopic data of the new compounds $\mathbf{4}$ and $\mathbf{6 a}-\mathbf{i}$ are available as supporting information available at (www.ias.ac.in/chemsci).

\section{Acknowledgments}

This work was partially supported by National Natural Science Foundation of China [No. 21172181, 
21372186, 81350110523, 81450110339 (The Research Fellowship for International Young Scientists from International (Regional) Cooperation and Exchange Program)], the key program from Natural Science Foundation of Chongqing (CSTC2012jjB10026), the Specialized Research Fund for the Doctoral Program of Higher Education of China (SRFDP 20110182110007).

\section{References}

1. Hopkins A L, Bickerton G R, Carruthers I M, Boyer S K, Rubin H and Overington J P 2011 Curr. Top. Med. Chem. 111292

2. Peng X M, Cai G X and Zhou C H 2013 Curr. Top. Med. Chem. 131963

3. Zhang L, Peng X M, Damu G L V, Geng R X and Zhou C H 2014 Med. Res. Rev. 34340

4. Peng X M, Damu G L V and Zhou C H 2013 Curr. Pharm. Des. 193884

5. Cui S F, Ren Y, Zhang S L, Peng X M, Damu G L V, Geng R X and Zhou C H 2013 Bioorg. Med. Chem. Lett. 233267

6. Yin B T, Yan C Y, Peng X M, Zhang S L, Rasheed S, Geng R X and Zhou C H 2014 Eur. J. Med. Chem. 71 148

7. Zhou C H and Wang Y 2012 Curr. Med. Chem. 19239

8. Ji H T, Zhang W N, Zhang M, Kudo M, Aoyama Y, Yoshida Y, Sheng C Q, Song Y L, Yang S, Zhou Y J, Lv $\mathrm{J}$ G and Zhu J J 2003 Med. Chem. 46474

9. Zhang H Z, Damu G L V, Cai G X and Zhou C H 2013 Eur. J. Med. Chem. 64329

10. Wang Y, Damu G L V, Lv J S, Geng R X, Yang D C and Zhou C H 2012 Bioorg. Med. Chem. Lett. 225363

11. Fang B, Zhou C H and Rao X C 2010 Eur. J. Med. Chem. 454388

12. Zhang S L, Chang J J, Damu G L V, Geng R X and Zhou C H 2013 Med. Chem. Comm. 4839

13. Ren Y, Zhang L, Zhou C H and Geng R X 2014 Med. Chem. 4640

14. Bhardwaj B, Kushwaha L and Kushwaha K 2014 Asian J. Biochem. Pharm. Res. 4164

15. Suma B V, Natesh N N and Madhavan V 2011 J. Chem. Pharm. Res. 3375

16. Piccionello A P and Guarcello A 2010 Curr. Bioact. Compd. 6266

17. Borowiecki P, Wawro A M, Winska P, Wielechowska M and Bretner M 2014 Eur. J. Med. Chem. 84364

18. Makowska M, Lukowska-Chojnacka E, Winska P, Kus A, Bilinska-Chomik A and Bretner M 2011 Mol. Cell. Biochem. 35691

19. Huang X H, Cheng C C, Fischmann T O, Duca J S, Richards M, Tadikonda P K, Reddy P A, Zhao L Y, Arshad S M, Parry D, Davis N, Seghezzi W, Wiswell D and Shipps G W 2013 Bioorg. Med. Chem. Lett. 232590

20. Fu J, Wang Y, Zhang X W, Mao W J, Zhang Z M and Zhu H L 2010 Bioorg. Med. Chem. 188457
21. Zhang S, Luo Y, He L Q, Liu Z J, Jiang A Q, Yang Y H and Zhu H L 2013 Bioorg. Med. Chem. 213723

22. Khabnadideh S, Rezaei Z, Pakshir K, Zomorodian K and Ghafari N 2012 Res. Pharm. Sci. 765

23. Patel P K and Patel P D 2010 Int. J. Chem. Tech. Res. 2 1147

24. Damu G L V, Cui S F, Peng X M, Wen Q M, Cai G $\mathrm{X}$ and Zhou C H 2014 Bioorg. Med. Chem. Lett. 24 3605

25. Shi Y, Zhou C H, Zhou X D, Geng R X and Ji Q G 2011 Acta Pharm. Sinica 46798 (in Chinese)

26. Ramalingam P, Ganapaty S and Rao C B 2010 Bioorg. Med. Chem. Lett. 20406

27. Kang I J, Wang L W, Yeh T K, Lee C, Lee Y C, Hsu S J, Wu Y S, Wang J C, Chao Y S, Yueh A and Chern J H 2010 Bioorg. Med. Chem. 186414

28. Lopez-Vallejo F, Castillo R, Yepez-Mulia L and Medina-Franco J L J 2011 Biomol. Screening 16 862

29. Korhonen J, Kuusisto A, Van B J, Patel J Z, Laitinen T, Navia-Paldanius D, Laitinen J T, Savinainen J R, Parkkari T and Nevalainen T J 2014 Bioorg. Med. Chem. 226694

30. Pavi K, Perkovi I, Cindri M, Pranji M, Martin-Kleiner I, Kralj M, Schols D, Hadjipavlou-Litina D, Katsori A M and Zorc B 2014 Eur. J. Med. Chem. 86502

31. Patel P D, Patel M R, Kocsis B, Kocsis E, Graham S M, Warren A R, Nicholson S M, Billack B, Fronczek F R and Talele T T 2010 Eur. J. Med. Chem. 452214

32. Yu G P, Xu L Z, Yi X, Bi W Z, Zhu Q and Zhai Z W 2009 J. Agric. Food Chem. 574854

33. Gan L L, Fang B and Zhou C H 2010 Bull. Korean Chem. Soc. 311

34. Perez E R, Loupy A, Liagre M, Plepis A M G and Cordeiro P J 2003 Tetrahedron 59865

35. Jacques J, Rajan S, Martin O and Sarel F M 2013 Tetrahedron Lett. 546923

36. Rahban M, Divsalar A, Saboury A A and Golestani A 2010 J. Phys. Chem. C 1145798

37. (a) Li X L and Hu Y J 2012 Biomacromolecules 13 873; (b) Wolfe A, Shimer G H and Meehan T 1987 Biochemistry 266392

38. Manna A and Chakravorti S 2015 Spectrochim. Acta A 150120

39. Zhang G W, Fu P, Wang L and Hu M M 2011 J. Agr. Food Chem. $\mathbf{5 9} 8944$

40. Awasthi S K, Mishra N, Dixit S K, Singh A, Yadav M, Yadav S S and Rathaur S 2009 J. Trop. Med. Hyg. 80 764

41. Sudhapriya N, Perumal $\mathrm{P} T$, Balachandran C, Ignacimuthu S and Sangeetha M 2014 Eur. J. Med. Chem. 83190

42. Shebl M and Khalil S M E 2015 Monatsh. Chem. 14615

43. Revankar H M, Kulkarni M V, Joshi S D and More U A 2013 Eur. J. Med. Chem. 70750

44. Nahid S, Soraya M F and Fahimeh K 2013 J. Photochem. Photobiol. B: Biology 12820

45. Zhang R F, Lu Y J, Zhang R R, Fu C J, Tang Z K and Zhang K 2013 J. Instrum. Anal. 32681 (in Chinese) 